\title{
The effectiveness and value of eculizumab and efgartigimod for generalized myasthenia gravis
}

\author{
A summary from the Institute for Clinical and Economic Review's New England \\ Comparative Effectiveness Public Advisory Council
}

Jeffrey A Tice, MD; Daniel R Touchette, PharmD, MA; Pei-Wen Lien, BPharm, MSc; Foluso Agboola, MBBS, MPH; Dmitriy Nikitin, MSPH; and Steven D Pearson, MD, MSC

Myasthenia gravis (MG) is an autoimmune disease that affects the neuromuscular junction, producing a characteristic finding of muscle weakness that worsens with repeated use ("fatigable weakness"). ${ }^{1}$ The prevalence in the United States is estimated to be between 14 and 20 per 100,000 people, ${ }^{2,3}$ and the annual incidence is approximately 2.2 per $100,000 .^{4}$ Approximately $85 \%$ of patients with MG test positive for the presence of antibodies to the acetylcholine receptor (AChR). ${ }^{5}$

With progressive disease, treatment typically includes high-dose corticosteroids combined with or followed by "steroid-sparing" immunosuppressive drugs (most commonly azathioprine or mycophenolate mofetil). The goal of therapy is to maintain the patient with minimal manifestations of disease (no symptoms or functional limitations from MG despite minimal weakness on examination) or better. ${ }^{6}$ Currently, it is estimated that about 20,000 patients with generalized MG are intolerant or have an inadequate response to conventional treatment options. ${ }^{7}$

In October 2017, the US Food and Drug Administration (FDA) approved eculizumab, a parenteral monoclonal antibody that inhibits complement cleavage, for generalized myasthenia gravis(gMG)patientswhoareanti-AChR antibody positive. Efgartigimod, a parenteral immunoglobulin fragment that targets the neonatal Fc receptor, is expected to receive FDA approval in December 2021.

The Institute for Clinical and Economic Review (ICER) conducted a systematic literature review and cost-effectiveness analysis to evaluate the health and economic outcomes of eculizumab and efgartigimod to treat gMG. Complete details of ICER's systematic literature search and protocol, as well as the methodology and model structure for the economic evaluation, are available on ICER's website.

In this report, we present the summary of our findings and highlights of the policy discussion with key stakeholders held at a public meeting of the New England Comparative Effectiveness Public Advisory Council on September 24, 2021. The full report is available on the ICER website at https://icer.org/wp-content/ uploads/2021/03/ICER_MyastheniaGravis_Final-Report_102021-1.pdf.

\section{Summary of Findings}

\section{CLINICAL EFFECTIVENESS}

In the phase 3 REGAIN trial, patients with anti-AChR antibody positive,

\section{Author affiliations}

Jeffrey A Tice, MD, Division of General Internal Medicine, University of California San Francisco. Daniel R Touchette, PharmD, MA, and Pei-Wen Lien, BPharm, MSc, University of Illinois at Chicago College of Pharmacy, Chicago, IL. Foluso Agboola, MBBS, MPH; Dmitriy Nikitin, MSPH; and Steven D Pearson, MD, MSC, Institute for Clinical and Economic Review (ICER), Boston, MA.

AUTHOR CORRESPONDENCE:

Jeffrey A Tice, jeff.tice@ucsf.edu

J Manag Care Spec Pharm. 2022;28(1):119-24

Copyright $\odot 2022$, Academy of Managed Care Pharmacy. All rights reserved.

"refractory" gMG who received eculizumab had significantly better improvement in MG activities of daily living (MG-ADL) score than those on placebo at 4 weeks and 8 weeks (Table 1), and the improvement was sustained at 26 weeks $(-4.2$ vs $-2.3, \quad \mathrm{P}=0.0058$; minimal clinically important difference $=2$ points). Similar patterns of improvement that favored eculizumab compared with 


\begin{tabular}{|c|c|c|c|c|c|}
\hline \multirow{2}{*}{$\begin{array}{l}\text { Intervention } \\
\text { (trial) }\end{array}$} & \multirow[b]{2}{*}{ Arms } & \multicolumn{2}{|c|}{$\triangle$ MG-ADL } & \multicolumn{2}{|c|}{$\triangle$ QMG } \\
\hline & & 4 weeks & 8 weeks & 4 weeks & 8 weeks \\
\hline \multirow{2}{*}{$\begin{array}{l}\text { culizumab } \\
\text { REGAIN) }\end{array}$} & Eculizumab & -3.5 & -3.7 & -3.3 & -4.0 \\
\hline & Placebo & -1.5 & -1.8 & -1.5 & -1.4 \\
\hline \multirow{2}{*}{$\begin{array}{l}\text { fgartigimod } \\
\text { ADAPT) }\end{array}$} & Efgartigimod & -4.6 & -2.2 & -6.2 & -2.9 \\
\hline & Placebo & -1.8 & -1.7 & -1.0 & -1.2 \\
\hline
\end{tabular}

Note: Numbers are digitized estimates.

$\Delta=$ change; $A C h R=$ acetylcholine receptor; $M G-A D L=$ Myasthenia Gravis Activities of Daily Living score; QMG = Quantitative Myasthenia Gravis score.

placebo were seen for the changes in the quantitative myasthenia gravis (QMG) score, myasthenia gravis composite (MGC) scale, and quality of life as assessed by the MG quality of life 15-item scale (MG-QOL-15). At week 26, the proportion of patients with minimal symptom expression (MG-ADL score of 0 or 1) was much greater in the eculizumab group (21.4\% vs $1.7 \%, P=0.0007) .{ }^{8}$ In the open label extension through 130 weeks of follow-up, the benefits were maintained and may have increased when compared with 26 weeks. ${ }^{9}$ There were no excess adverse events in the trial, although more patients in the eculizumab group stopped treatment due to adverse events, and eculizumab carries a black box warning for meningococcal infections.

The phase 3 ADAPT trial evaluating efgartigimod was conducted in a broader population of gMG patients who were not required to be antiAChR antibody positive or refractory to standard therapies. However, patients who were anti-AChR antibody negative were a small proportion of trial participants, and an a priori decision was made to base the primary outcome only on findings from antiAChR antibody positive patients. In this population, patients who received efgartigimod did significantly better than those who received placebo on the primary outcome (significant improvement in MG-ADL during the first treatment cycle (MG-ADL responders $68 \%$ vs $30 \%, \mathrm{P}<0.0001){ }^{10}$

In addition, at week 4 , the efgartigimod group had a clinically and statistically significantly greater reduction in the 30-point MG-QOL$15 \mathrm{r}$ scale $(-7.3$ vs -2.3 points, $P<0.05)$. The improvements in the efgartigimod group compared with the placebo group were greater at 4 weeks than at 8 weeks (Table 1), reflecting the unusual dosing schedule used in this trial. Patients were treated weekly for 4 weeks, and then treatment was held for a minimum of 4 weeks. Patients received their second treatment cycle at week 8 or later only when they no longer had a clinically meaningful improvement on the MG-ADL. Thus, many patients were back near baseline at 8 weeks, having not been treated for 4 weeks.

In the exploratory analyses performed with data on outcomes in the anti-AChR antibody negative population, patients in the efgartigimod and placebo groups had similar response rates on the MG-ADL $(68 \%$ eculizumab vs $63 \%$ placebo, $P=N R$ ). Adverse events were not more common with efgartigimod, but there are long-term concerns about infections with the lowering of immunoglobulin G levels.

\section{LIMITATIONS OF THE CLINICAL EVIDENCE}

First, it is not clear if or when to stop or to taper either of the drugs once initiated, other than for patients who do not respond. Second, the target population for treatment is uncertain. Eculizumab was studied only in patients' refractory to standard therapies, but the FDA label does not specify limiting use to refractory patients. Efgartigimod's pivotal trial included anti-AChR antibody positive and negative patients, but the primary outcome was in antibody positive patients. It is unclear if it should be used to treat antibody negative patients.

For efgartigimod, the primary uncertainty is the whether the dosing regimen used in the ADAPT trial will be included in the FDA label and, ultimately, how the dosing will be managed in real-world clinical practice. In the ADAPT trial, subsequent cycles were started once patients lost clinical benefits. But clinical experts have advised that it seems likely that, in routine practice, patients and clinicians will not want to wait until the benefits have receded before starting another round of therapy. Finally, there are limited data on long-term safety for efgartigimod.

\section{LONG-TERM COST-EFFECTIVENESS}

The economic model evaluated the cost effectiveness of eculizumab plus conventional therapy vs conventional therapy alone in patients with refractory anti-AChR antibody positive gMG as defined in the REGAIN trial and efgartigimod plus conventional therapy vs conventional therapy alone in patients with gMG, including those with or without anti-AChR antibodies. The analyses were framed to evaluate results over a 2-year time 


\section{TABLE 2}

\section{Model Results for Eculizumab and Efgartigimod Compared with Conventional Therapy}

\begin{tabular}{|c|c|c|c|c|c|}
\hline \multirow[b]{2}{*}{ Deterministic analysis } & \multirow[b]{2}{*}{$\begin{array}{c}\text { Total costs } \\
\$\end{array}$} & \multirow[b]{2}{*}{ QALYs } & \multirow[b]{2}{*}{ LYs } & \multicolumn{2}{|c|}{ Incremental results } \\
\hline & & & & $\begin{array}{c}\text { Cost/QALY } \\
\text { gained } \\
\text { (same as cost } \\
\text { per evLYG) }\end{array}$ & $\begin{array}{l}\text { Cost/LY } \\
\text { gained }^{\text {a }}\end{array}$ \\
\hline \multicolumn{6}{|c|}{ Comparison of eculizumab to conventional therapy } \\
\hline Eculizumab plus CT & 855,400 & 1.13 & 1.93 & $5,210,000$ & N/A \\
\hline CT alone & 95,500 & 0.98 & 1.93 & - & - \\
\hline \multicolumn{6}{|c|}{ Comparison of efgartigimod to conventional therapy } \\
\hline Efgartigimod plus $\mathrm{CT}^{\mathrm{b}}$ & 692,700 & 1.27 & 1.93 & $2,076,000$ & N/A \\
\hline CT alone & 94,800 & 0.98 & 1.93 & - & - \\
\hline
\end{tabular}

${ }^{a}$ There were no differences in survival. Cost per life-year gained could not be calculated, whereas cost per evLYG is equal to the cost per QALY gained.

${ }^{b}$ Efgartigimod was evaluated using a placeholder price.

evLYG = equal value of life-years gained; $C T=$ conventional therapy; $L Y=$ life-year; $N / A=$ not applicable; QALY=quality-adjusted life-year.

horizon, taking a health system perspective. A detailed description of the model can be found in the full report (https://icer.org/wp-content/ uploads/2021/03/ICER_MyastheniaGravis_Final-Report_102021-1.pdf).

Treatment with eclulizumab and efgartigimod were not assumed to prolong the life of patients with gMG, thus cost-effectiveness findings of incremental cost per quality-adjusted life-year (QALY) and incremental cost per equal value of life-years gained (evLYG) are the same. For eculizumab, with an estimated annual cost of $\$ 653,100$, the incremental costeffectiveness ratio was $\$ 5,210,000$ per QALY/evLYG (Table 2). For analyses of efgartigimod, we used a placeholder price of $\$ 418,400$, which is the midpoint of the potential price range cited by the manufacturer." At this estimated price, efgartigimod has an the incremental cost per QALY/evLYG of $\$ 2,076,000$ (Table 2).

The economic model was most sensitive to inputs such as the quality of life improvement assigned to improved and unimproved MG health states and the proportion of patients achieving at least a 3-point reduction in the QMG. However, despite the large impact of changing these inputs on the results, the incremental cost-effectiveness ratio was never less than \$3.8 million per QALY/evLYG for eculizumab and \$1.7 million per QALY/ evLYG for efgartigimod. In probabilistic sensitivity analyses, neither drug was ever found to reach cost-effectiveness at the $\$ 150,000$ per QALY/ evLYG threshold.

\section{LIMITATIONS OF THE COST- EFFECTIVENESS MODEL}

Both drugs were evaluated in single, small phase 3 randomized trials of limited duration. Thus, there remains substantial uncertainty in the true relative effectiveness of treatments. The longer-term effects of the treatments on clinical outcomes, including reductions in concurrent therapies such as oral corticosteroids and immunosuppressive treatments, have not been well described. There were very limited studies evaluating the association between change in QMG or MG-ADL and the overall costs of MG treatment. There were insufficient data to support modeling from a societal perspective, although the analysis would be informative given the degree of disability associated with gMG. Finally, there remains uncertainty surrounding the price and the real-world dosing frequency of efgartigimod.

\section{Policy Discussion}

The New England Comparative Effectiveness Public Advisory Council (CEPAC) is one of the independent appraisal committees convened by ICER to engage in the public deliberation of the evidence on clinical and cost-effectiveness of health care interventions. The New England CEPAC is composed of medical evidence experts, including practicing clinicians, methodologists, and leaders in patient engagement and advocacy. Their deliberation includes input from clinical experts and patient representatives specific to the condition under review, as well as formal comments from manufacturers and the public. In addition, a policy roundtable concludes each meeting during which representatives from insurers and manufacturers join clinical experts and patient representatives to discuss how best to apply the findings of the evidence to clinical practice, insurance coverage, and pricing negotiations.

The ICER report on new therapies for gMG was the subject of a New England CEPAC meeting on September 24, 2021. Following the discussion, the New England CEPAC panel members deliberated on key questions raised by ICER's report. For the population of patients with gMG who test positive for anti-AChR antibodies, the panel voted as follows: (1) 11-0 that the evidence is adequate to demonstrate the superiority of eculizumab added to conventional therapy vs conventional 


\section{TABLE 3 Votes on Contextual Considerations and Potential Other Benefits or Disadvantages for Any Effective Treatment for Myasthenia Gravis}

When making judgments of overall long-term value for money, what is the relative priority that should be given to any effective treatment for gMG, on the basis of the following contextual considerations?

\begin{tabular}{l|c|c|c|c}
\multicolumn{1}{c|}{ Contextual consideration } & Very low priority & Low priority & Average priority & $\begin{array}{c}\text { Very high } \\
\text { priority }\end{array}$ \\
\hline $\begin{array}{l}\text { Acuity of need for treatment of individual patients } \\
\text { based on the severity of the condition being treated }\end{array}$ & 0 & 2 & 2 & 7 \\
\hline $\begin{array}{l}\text { Magnitude of the lifetime impact on individual patients } \\
\text { of the condition being treated }\end{array}$ & 0 & 0 & 1 & 7 \\
\hline
\end{tabular}

gMG = generalized myasthenia gravis.

\section{TABLE 4 Votes on Contextual Considerations and Potential Other Benefits or Disadvantages for Eculizumab vs Usual Care}

What are the relative effects of eculizumab vs conventional therapy on the following outcomes that inform judgment of the overall long-term value for money of eculizumab?

\begin{tabular}{|c|c|c|c|c|c|}
\hline Potential other benefit or disadvantage & $\begin{array}{c}\text { Major negative } \\
\text { effect }\end{array}$ & $\begin{array}{c}\text { Minor negative } \\
\text { effect }\end{array}$ & No difference & $\begin{array}{l}\text { Minor positive } \\
\text { effect }\end{array}$ & $\begin{array}{l}\text { Major positive } \\
\text { effect }\end{array}$ \\
\hline $\begin{array}{l}\text { Patients' ability to achieve major life goals related to } \\
\text { education, work, or family life }\end{array}$ & 0 & 0 & 0 & 8 & 3 \\
\hline $\begin{array}{l}\text { Caregivers' quality of life and/or ability to achieve major } \\
\text { life goals related to education, work, or family life }\end{array}$ & 0 & 0 & 1 & 8 & 2 \\
\hline Society's goal of reducing health inequities & 3 & 0 & 7 & 1 & 0 \\
\hline
\end{tabular}

therapy alone; (2) 10-1 that the evidence is adequate to demonstrate the superiority of efgartigimod added to conventional therapy vs conventional therapy alone; and (3) 11-0 that the clinical evidence is inadequate to distinguish the net health benefit of eculizumab from that of efgartigimod. The panel also voted 11-0 and 10-1 that the clinical evidence was inadequate to distinguish the net health benefit of eculizumab and efgartigimod from intravenous immunoglobulin and from rituximab, respectively.

Notably, for the population of patients with gMG who test negative for anti-AChR antibodies, the panel voted 11-0 that the evidence is inadequate to demonstrate that the net health benefit of efgartigimod is superior to that of conventional therapy because of the limited data available for this population.

The CEPAC panel also voted on "other potential benefits" and "contextual considerations" as part of a process intended to signal to policymakers whether there are important considerations when making judgments about long-term value for money not fully represented in analyses of clinical and/or cost-effectiveness. The results of these votes are shown in Tables 3-5. They highlight several factors beyond the results of cost-effectiveness modeling that the CEPAC panel felt were particularly important for judgments of the overall long-term value for money of treatments for gMG.

The final votes on the long-term value for money reflect the integration of the contextual considerations, other potential benefits and the cost effectiveness results. The 11 CEPAC panel members voted that, for patients with "refractory" gMG who test positive for anti-AChR antibodies, the long-term value for money of eculizumab added to conventional therapy compared with conventional therapy alone was low (10 votes) or intermediate (1 vote). For patients with gMG, assuming the placeholder price used in this review, the long-term value for money of efgartigimod added to conventional therapy compared with conventional therapy alone was deemed low (11 votes).

The policy roundtable discussion explored how best to translate the evidence and additional considerations into clinical practice and into pricing and insurance coverage policies. The full set of policy recommendations can be 


\section{TABLE 5 Votes on Contextual Considerations and Potential Other Benefits or Disadvantages for Efgartigimod vs Usual Care}

\begin{tabular}{|c|c|c|c|c|c|}
\hline \multicolumn{6}{|c|}{$\begin{array}{l}\text { What are the relative effects of efgartigimod vs conventional therapy on the following outcomes that inform judgment of the overall } \\
\text { long-term value for money of efgartigimod? }\end{array}$} \\
\hline Potential other benefit or disadvantage & $\begin{array}{c}\text { Major negative } \\
\text { effect }\end{array}$ & $\begin{array}{c}\text { Minor negative } \\
\text { effect }\end{array}$ & No difference & $\begin{array}{l}\text { Minor positive } \\
\text { effect }\end{array}$ & $\begin{array}{l}\text { Major positive } \\
\text { effect }\end{array}$ \\
\hline $\begin{array}{l}\text { Patients' ability to achieve major life goals related to } \\
\text { education, work, or family life }\end{array}$ & 0 & 0 & 1 & 10 & 0 \\
\hline $\begin{array}{l}\text { Caregivers' quality of life and/or ability to achieve major } \\
\text { life goals related to education, work, or family life }\end{array}$ & 0 & 0 & 2 & 9 & 0 \\
\hline Society's goal of reducing health inequities & 3 & 0 & 7 & 1 & 0 \\
\hline
\end{tabular}

found in the Final Evidence Report on the ICER website: https://icer.org/ assessment/myasthenia-gravis/.

Several key policy recommendations for the new treatments for gMG follow:

Recommendation: Safe and effective treatment for gMG remains a significant unmet health care need. Efforts are needed to ensure that new therapies for myasthenia gravis, such as eculizumab and efgartigimod, improve the health of patients and families and do not aggravate existing health inequities. Clinical experts and patients highlighted that the high cost of new therapies may worsen disparities in accessing care. This may be due to lack of health insurance, which limits access to specialists and the new therapies that they prescribe, or high deductible payments even for those with insurance may result in steep out-of-pocket costs.

Recommendation: Payers should use the FDA label as the guide to coverage policy and engage clinical experts and diverse patient representatives in considering how to address coverage issues for which there is limited or no evidence at the current time. Given the significant uncertainty that remains about the new therapies for $\mathrm{gMG}$, it is reasonable for payers to use prior authorization as a component of coverage. Prior authorization criteria should be based on the FDA label, clinical trial eligibility criteria, specialty society guidelines, and input from clinical experts and patient groups. The process for authorization should be clear and efficient for providers and patients. General fair access design criteria have been described in ICER's white paper on fair drug coverage (https://34eyj51jerf417itp82 ufdoe-wpengine.netdna-ssl. com/w p-content/uploads/ 2020/11/Cornerstones-of-FairDrug-Coverage- -September-282020-corrections-1-5-21.pdf), with perspectives on specific elements of coverage criteria for new therapies for gMG provided in the section on drug-specific considerations.

Recommendation: The price for eculizumab is extremely high and is distinctive for the amount by which it exceeds the price needed to reach traditional cost-effectiveness thresholds in the United States. Eculizumab was first approved for paroxysmal nocturnal hemoglobinuria and hemolytic uremic syndrome, ultra-rare conditions with a cumulative prevalence of less than 4 per million. The population with gMG is $14-20$ per 100,000 , and if only $15 \%$ of this population is considered to have refractory gMG, the population now eligible for treatment with eculizumab is more than 7 times as large as when the drug was first approved, yet the price has not come down. There is no excuse for this level of pricing, and it should not be used as a benchmark or standard for future therapies in this clinical area.

\section{DISCLOSURES}

Funding for this summary was contributed by Arnold Ventures, The Donaghue Foundation, Harvard Pilgrim Health Care, and Kaiser Foundation Health Plan to the Institute for Clinical and Economic Review (ICER), an independent organization that evaluates the evidence on the value of health care interventions.

ICER's annual policy summit is supported by dues from AbbVie, America's Health Insurance Plans, Anthem, Alnylam, AstraZeneca, Biogen, Blue Shield of CA, Boehringer-Ingelheim, Cambia Health Services, CVS, Editas, Evolve Pharmacy, Express Scripts, Genentech/Roche, GlaxoSmithKline, Harvard Pilgrim, Health Care Service Corporation, HealthFirst, Health Partners, Humana, Johnson \& Johnson (Janssen), Kaiser Permanente, LEO Pharma, Mallinckrodt, Merck, Novartis, National Pharmaceutical Council, Pfizer, Premera, Prime Therapeutics, Regeneron, Sanofi, Sun Life Financial, uniQure, and United Healthcare.

Agboola, Nikitin, and Pearson are employed by ICER. Through their affiliated institutions, Tice, Touchette, and Lien received funding from ICER for the work described in this summary. 


\section{ACKNOWLEDGMENTS}

The authors thank John Campbell, Ashton Moradi, Mrinmayee Joshi, Zaid Yousif, and Shani Patel for their contributions to this report.

\section{REFERENCES}

1. Gilhus NE. Myasthenia gravis. New Engl J Med. 2016;375(26):2570-81.

2. Carr AS, Cardwell CR, McCarron PO, McConville J. A systematic review of population based epidemiological studies in myasthenia gravis. BMC Neurol. 2010;10:46.

3. Howard J Jr. Myasthenia gravis clinical overview. 2015. Accessed May 5, 2021. https://myasthenia.org/Professionals/ Clinical-Overview-of-MG

4. Hendricks TM, Bhatti MT, Hodge DO, Chen JJ. Incidence, epidemiology, and transformation of ocular myasthenia gravis: a population-based study. Am J Ophthalmol. 2019;205:99-105.
5. Gilhus NE, Verschuuren JJ. Myasthenia gravis: subgroup classification and therapeutic strategies. Lancet Neurol. 2015;14(10):1023-36.

6. Narayanaswami P, Sanders DB, Wolfe $\mathrm{G}$, et al. International consensus guidance for management of myasthenia gravis: 2020 update. Neurology. 2021;96(3):114-22.

7. Phillips G. argenx response to ICER's draft evidence report assessing treatments for myasthenia gravis. In: ICER Myathenia Gravis Public Comments Folio. August 18, 2020. Pages 6-10. Accessed December 15, 2021. https:// icer.org/wp-content/uploads/2021/03/ ICER Myasthenia-Gravis PublicComment-Folio 091021.pdf

8. Vissing J, Jacob S, Fujita KP, et al. 'Minimal symptom expression' in patients with acetylcholine receptor antibody-positive refractory generalized myasthenia gravis treated with eculizumab. J Neurol. 2020;267(7):1991-2001.
9. Mantegazza R, Wolfe GI, Muppidi S, et al. Post-intervention status in patients with refractory myasthenia gravis treated with eculizumab during REGAIN and its open-label extension. Neurology. 2021;96(4):e610-e618.

10. Howard J, Bril V, Vu T, et al. Safety, efficacy, and tolerability of efgartigimod in patients with generalised myasthenia gravis (ADAPT): a multicentre, randomised, placebo-controlled, phase 3 trial. Lancet Neurol. 2021;20(7):526-36.

11. The Motley Fool. argenx SE (ARGX) Q3 2020 earnings call transcript. October 22, 2020. Accessed June 7, 2021. https://www.fool.com/earnings/ call-transcripts/2020/10/22/argenx-seargx-q3-2020-earnings-call-transcript/ 